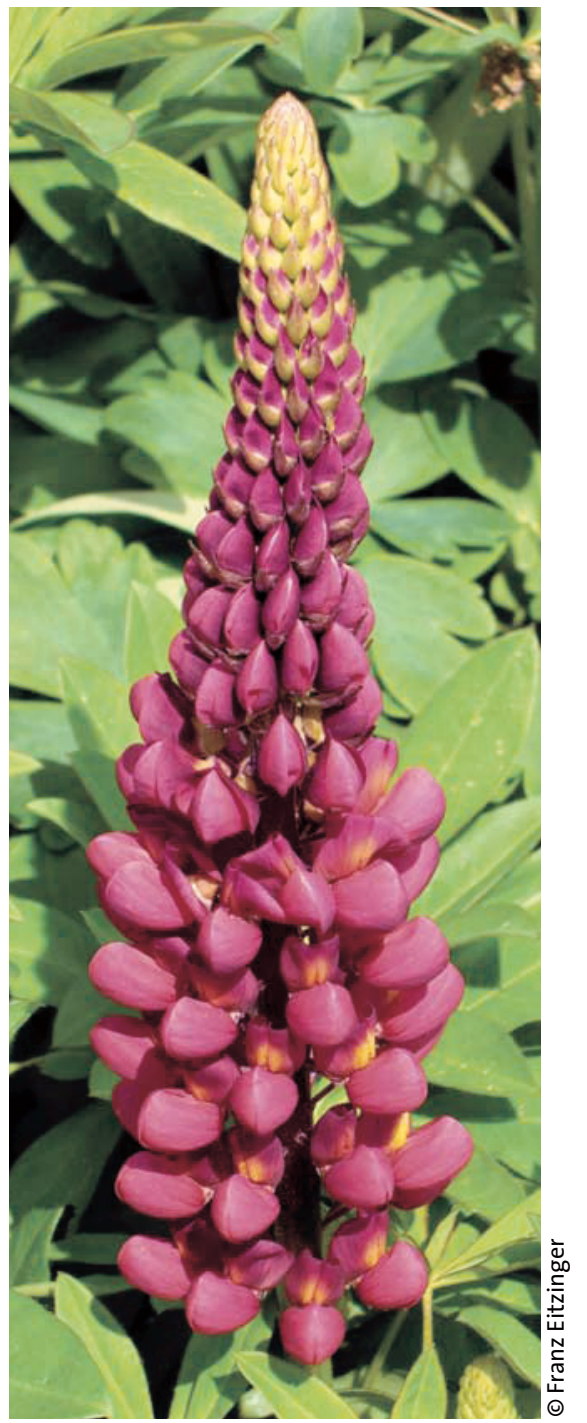

Die Lupine (hier Lupinus polyphyllus) ist ein Neophyt, der Anfang des 19. Jahrhunderts als Gartenpflanze aus Nordamerika nach Europa eingeführt wurde, wo er inzwischen auch wild vorkommt.

Impressum ,Der ÅDA informiert"

Verantwortlich für den Inhalt

Prof. Dr. Ludger Klimek, Wiesbaden

\section{Redaktion}

Markus Seidl

Verlag

(c) Urban \& Vogel GmbH, München

\section{ÄDA-Geschäftsstelle}

Service Systems, Dreieich

Tel.: (o 61 03) 62273

Fax: (o 6103$) 697019$

E-Mail:info@aeda.de

Internet: www.aeda.de

\title{
Lupinenmehlallergie ist im Kommen
}

\author{
Der Trend zur gesundheitsbewussten Ernährung bringt auch neue \\ Allergien mit sich: Dazu gehört die Lupinenmehlallergie. Das \\ französische Allergy Vigilance Network hat jetzt in einer großen \\ Untersuchung Daten zur Prävalenz erhoben.
}

D ie Lupine ist eine Hülsenfrucht, deren Mehl sich als Backzusatz eignet, der die Farbe, Beschaffenheit und biologische Wertigkeit des Produkts beeinflusst. Bei der Herstellung verschiedenen Backwerks wie Brot, Pizza und Kleingebäck wird Lupinenmehl daher heute gerne dem Weizenmehl beigemischt. Seine Menge kann dabei bis zu $10 \%$ der Weizenmehlmenge betragen. Auch Fertigsuppen können Lupinenmehl enthalten, und manche Weine werden mit Lupinenmehl geklärt. Diätetisch dient es nicht zuletzt als alternatives Kohlehydrat.

Wie sich zeigt, ist jedoch auch das allergene Potenzial nicht zu unterschätzen. Allergische Reaktionen gegen Lupinenmehl sind in unterschiedlicher klinischer Ausprägung bekannt, darunter auch schwere Anaphylaxien. Ausgelöst werden die Reaktionen meist auf nutritivem Weg - einige $100 \mathrm{mg}$ können bereits ausreichen -, selten auf inhalativem Weg im Zusammenhang mit beruflicher Exposition. Zu beachten ist, dass dieses Allergen wärmeresistent ist und seine Allergenität auch nach thermischen Aufbereitungsprozessen beibehält.

Unabhängig von der Art der Exposition ist die Sensibilisierung gegen Lupinenmehl wohl selten eine Primärreaktion. Vielmehr geht man davon aus, dass sie sekundär, infolge einer Kreuzallergie gegen ein anderes Antigen, zustande kommt. Eine Kreuzreaktion gegen Lupinenmehl wurde beispielsweise in $\mathrm{Zu}$ sammenhang mit einer Lupinenpollinosis beobachtet, insbesondere aber bei Erdnussallergie. Das mag nicht überraschen: Wie der englische Name der in Südamerika beheimateten Erdnuss „peanut“, also „Erbsennuss“ - andeutet, gehört sie wie die Lupine zur Familie der Hülsenfrüchte.
Untersuchungen zur Prävalenz der Lupinenmehlallergie an einem großen Patientenkollektiv standen jedoch bislang noch aus. Um hier einen Überblick zu gewinnen, führten die 88 französischen und belgischen Allergologen des Allergy Vigilance Network bei jedem ihrer Patienten, bei dem eine Allergiediagnsotik erforderlich war, auch einen Pricktest mit Lupinenmehlextrakt durch. Untersucht wurden 2.680 Kinder und 2.686 Erwachsene. Das gesamte Kollektiv wurde in vier Gruppen unterteilt: Patienten ohne Sensibilisierung, Patienten mit Nachweis von spezifischem IgE, aber ohne klinische Symptome, Patienten mit altueller Allergie und Patienten mit Erdnussallergie.

Die Auswertung des Kinderkollektivs ergab: Bei den Nicht-Atopikern konnte lediglich in $0,2 \%$ der Fälle eine Lupinenmehlsensibilisierung nachgewiesen werden, bei den Atopikern in 2\%, bei den Allergiekranken in 3\% und bei bei den Erdnussallergikern in $17 \%$ der Fälle. Für die erwachsenen Patienten lauteten die entsprechenden Zahlen 1\%, $2 \%, 4 \%$ sowie $15 \%$.

\section{Fazit}

Die Häufigkeit der Lupinenmehlsensibilisierung zwingt dazu, dieses Allergen routinemäßig ins diagnostische Kalkül einzubeziehen. Besondere Aufmerksamkeit ist Patienten mit bekannter Erdnussallergie zu widmen.

\section{Dr. Dieter Bruchhausen, Wuppertal}

\section{Literatur}

Gayraud J, Mairesse M, Fontaine JF, Thillay A, Leduc V, Rancé F, Parisot L, Moneret-Vautrin DA. The prevalence of sensitization to lupin flour in France and Belgium: a prospective study in 5,366 patients, by the Allergy Vigilance Network. Eur Ann Allergy Clin Immunol 2009; 41: 17-22 